
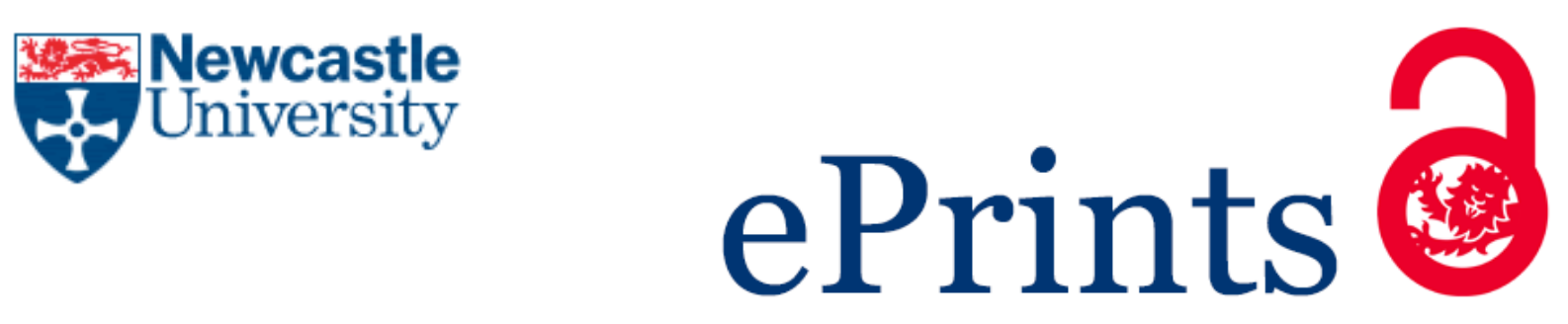

Nakamura S, Mitomo H, Aizawa M, Tani T, Matsuo Y, Niikura K, Pike AR, Naya N, Shishido A, ljiro K.

DNA Brush-Directed Vertical Alignment of Extensive Gold Nanorod Arrays with Controlled Density.

ACS Omega 2017, 2(5), 2208-2213.

\title{
Copyright:
}

ACS AuthorChoice - This is an open access article published under an ACS AuthorChoice License, which permits copying and redistribution of the article or any adaptations for non-commercial purposes.

DOI link to article:

https://doi.org/10.1021/acsomega.7b00303

Date deposited:

$20 / 10 / 2017$

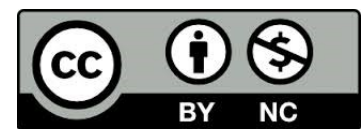

This work is licensed under a Creative Commons Attribution-NonCommercial 3.0 Unported License 


\section{DNA Brush-Directed Vertical Alignment of Extensive Gold Nanorod Arrays with Controlled Density}

Satoshi Nakamura, ${ }^{\dagger}$ Hideyuki Mitomo, ${ }^{*},{ }^{\ddagger} \S_{\odot}$ Miho Aizawa, ${ }^{\|}$Takeharu Tani, ${ }^{\perp}$ Yasutaka Matsuo,

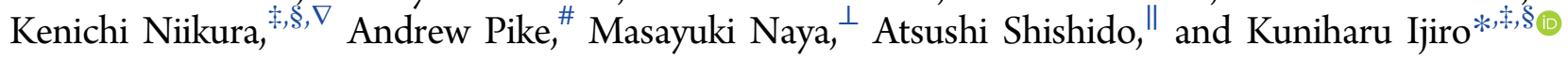

${ }^{\dagger}$ Graduate School of Chemical Sciences and Engineering, Hokkaido University, Sapporo 060-8628, Japan

${ }^{\ddagger}$ Research Institute for Electronic Science and ${ }^{\S}$ Global Station for Soft Matter, Global Institution for Collaborative Research and Education (GI-CoRE), Hokkaido University, Sapporo 001-0021, Japan

"Laboratory for Chemistry and Life Science, Institute of Innovative Research, Tokyo Institute of Technology, Yokohama 226-8503, Japan

${ }^{\perp}$ FUJIFILM Corporation, Kanagawa 258-8577, Japan

${ }^{\#}$ School of Chemistry, Newcastle University, Bedson Building, Newcastle upon Tyne NE1 7RU, U.K.

Supporting Information

ABSTRACT: Control over the orientation of metal nanorods is important for both fundamental and applied research. We show that gold nanorods (GNRs) can be aligned in a single direction by adsorbing positively charged GNRs onto a double-strand DNA-grafted substrate through electrostatic interaction. The ordered structure can be optimized by controlling the density of the positive charges on the surface of the GNRs. We found, in agreement with the results of theoretical simulation, that the resultant structure exhibits plasmonic properties that are dependent on the GNR orientation relative to the direction of an oscillating electric field. Our approach provides new insights into the polymer-assisted self-assembly of rod-shaped nanoparticles utilizing electrostatic interactions.

\section{INTRODUCTION}

Metal nanoparticles have an array of attractive electronic, optical, and thermal properties, which have a variety of applications in sensing, ${ }^{1-3}$ drug delivery, ${ }^{4,5}$ and catalysis. ${ }^{6,7}$ To harness these unique properties, their self-assembly into ordered structures is of strategic importance. ${ }^{8-12}$ Recently, selfassemblies of rod-shaped nanoparticles have been shown to display unique shape-dependent properties and functionalities. ${ }^{13,14}$ Examples of self-assembly techniques include dropcasting $^{15,16}$ and external field-directed assembly. ${ }^{17-20}$ Ordered structures of rod-shaped nanoparticles prepared by these previous techniques, however, are dotted and of microscale dimensions, as the nanoparticle assemblies are created through the drying process. The self-assembly of rod-shaped nanoparticles over a large area, with controlled density, via the drying process remains challenging due to thermal fluctuations and diffusion; nevertheless, the ability to control the orientation of anisotropic nanorods, in particular, is essential for the assembly of extensive ordered structures. Interfacial assembly $^{21,22}$ would enable orientation control of rod-shaped nanoparticles with controlled density, but the resultant structure needs to be transferred to a substrate for its application to devices. Recently, lithographically patterned templates allow selective and precise placement of nano- particles, providing ordered arrays. However, these lithographic nanotemplate-assisted techniques ${ }^{23,24}$ are unsuitable for extensive ordering of rod-shaped nanoparticles due to cost and time constraints.

Specifically designed molecules within self-assembled monolayers, liquid crystal molecules, and lipid bilayers can be more easily organized, with controlled orientation. In macromolecular chemistry, charged polymer chains condensed in a confined area tend to orient perpendicularly to the grafted plane, as interactions between adjacent molecules, including osmotic pressure and excluded volume effects, force them to stretch. $^{25-27}$ These surface-confined polymer chains are called "charged polymer brushes". Inspired by this "brush"-like configuration, we speculated that a negatively charged polymer brush could be used to control the orientation of positively charged rod-shaped nanoparticles through simple electrostatics. Although there are some reports on nanoparticle attachment on polymer brushes, no one has reported the vertical alignment of rod-shaped nanoparticles. ${ }^{28,29}$ Thus, we aimed to align positively charged gold nanorods (GNRs) vertically using

Received: March 14, 2017

Accepted: May 10, 2017

Published: May 19, 2017 
polymer brush-assisted self-assembly, with the assistance of a polymer brush composed of double-strand DNA (dsDNA), which is a stiff anionic polymer with a persistent length of ca. 50 $\mathrm{nm}$ (Scheme 1).

Scheme 1. Vertical Assembly of GNRs with the Assistance of a dsDNA Brush

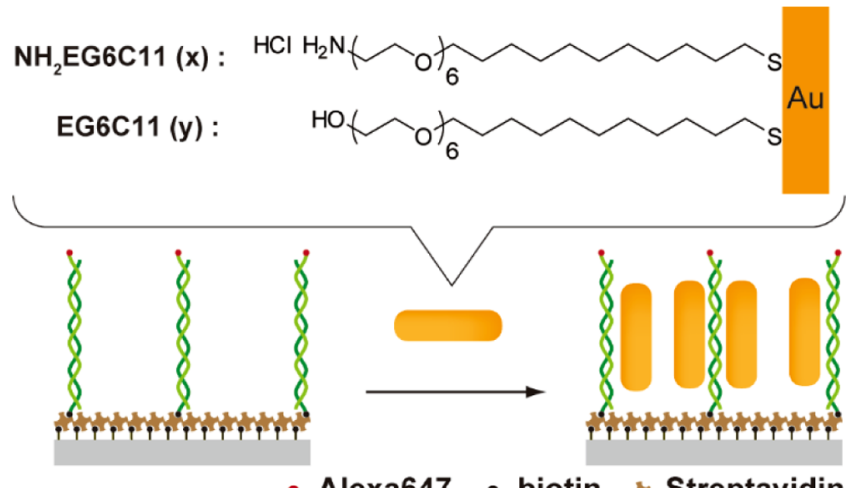

- Alexa647 - biotin + Streptavidin

\section{RESULTS AND DISCUSSION}

First, we prepared DNA brushes. We synthesized 148 bp dsDNA with biotin and fluorophore (Alexa 647) modifications at the $5^{\prime}$ ends of the complementary strands using a standard polymerase chain reaction (PCR) method. The biotinylated end was designed to allow facile immobilization on a streptavidin surface, and the Alexa 647 dye provided a spectroscopic tag to allow monitoring of brush formation. The length of the DNA is consistent with its persistent length, and it is expected to behave as a stiff brush, which is important for our purpose. We attached the DNA to a streptavidinfunctionalized substrate via the commonly exploited streptavidin-biotin interaction. We prepared the DNA brush on the inside surface of a glass cuvette, with an optical path length of 1 $\mathrm{mm}$. The density of the DNA brush surface coverage was derived from the fluorescence intensity of the Alexa 647 fluorophore by comparison to known amounts of Alexa 647labeled DNA in a non-streptavidin-modified cuvette. The density of the DNA brush was evaluated as 1200 chains $/ \mu \mathrm{m}^{2}$, corresponding to an average interchain distance of $31 \mathrm{~nm}$. This value is reasonable when compared to that in the results from the previous report. ${ }^{25}$ The average interchain distance of the DNA brush was calculated on the basis of the assumption that fully stretched DNA chains are arranged in a hexagonal pattern.

Second, we prepared GNRs $(34 \mathrm{~nm} \times 10 \mathrm{~nm})$ modified with a cationic alkanethiol ligand $\left(\mathrm{NH}_{2} \mathrm{EG} 6 \mathrm{C} 11\right.$, see Scheme 1$)$ on the basis of a previously reported procedure (see Supporting Information (SI), Figure S1). ${ }^{30}$ The GNRs exhibited two plasmonic modes. One is transverse localized surface plasmon resonance (TLSPR), around $510 \mathrm{~nm}$, excited by the oscillation of electrons along the major axis of the GNRs. The other is longitudinal localized plasmon resonance (LLSPR), around 760 $\mathrm{nm}$, excited by the oscillation of electrons along the minor axis of the GNRs. Optical properties of the GNRs were determined by their orientation relative to the direction of the oscillating electric field. Therefore, we could evaluate the orientation of the GNRs adsorbed onto the DNA brush by measuring the extinction spectrum. ${ }^{31}$ In the extinction spectrum analysis, unless otherwise stated, the direction of the incident light was perpendicular to the plane containing the adsorbed GNRs.
Therefore, when the GNRs align vertically, only the TLSPR should be excited. In contrast, when the GNR orientation is random, both TLSPR and LLSPR excitation should be observed.

The GNRs modified with the cationic ligand were adsorbed onto the DNA brush, and the extinction spectrum was recorded using nonpolarized light in a buffer solution (Figure S2). As a result, the extinction spectrum showed a strong peak for LLSPR at $920 \mathrm{~nm}$, indicating that the GNR orientation was random. However, we discovered that the intensity of the peak for LLSPR decreased under a high salt concentration $(2 \mathrm{M} \mathrm{NaCl})$ (Figure S2). Considering that electrostatic interaction is weakened in the presence of salt, this result suggests that the intensity of the electrostatic attraction between the DNA brushes and the GNRs influences the orientation of the adsorbed GNRs.

We further investigated the effect of electrostatic attraction intensity on nanorod orientation by tuning the density of the cationic ligand on the surface of the GNRs. The GNRs were modified with a cationic ligand and nonionic ligand (EG6C11, see Scheme 1) in various ratios. We confirmed that the $\zeta$ potential of the GNRs increased in proportion to the ratio of the cationic ligand (Figure S3). Hereafter, we denote GNRs modified with cationic and nonionic ligands in a ratio of $x: y$ as GNR $(x: y)$. The mixed ligand GNRs were adsorbed onto the DNA brush, and the extinction spectra were again recorded with nonpolarized light. We found that as the proportion of the cationic ligand decreased, the intensity of the peak for LLSPR also decreased (Figure 1). This result suggests that GNRs align

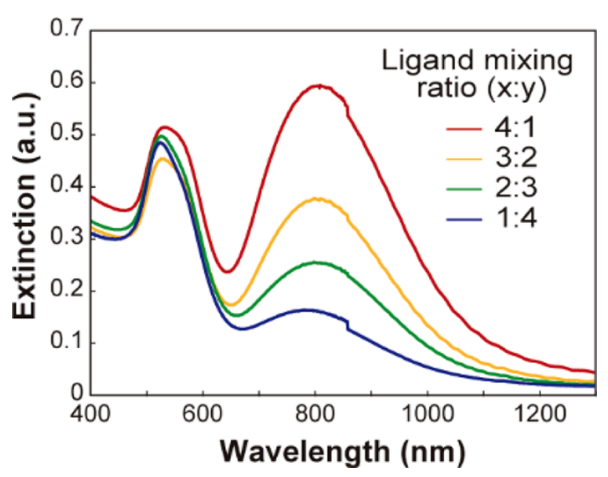

Figure 1. Extinction spectra of GNRs adsorbed on a DNA brush in 10 $\mathrm{mM}$ Tris $-\mathrm{HCl}$ buffer $(\mathrm{pH}=7.7)$. The various mixing ratios were used as shown. The adsorption time was $60 \mathrm{~min}$ in all cases.

mostly in a vertical direction when the electrostatic attraction is weak. However, it is still possible that the decrease in LLSPR intensity was caused by the plasmonic coupling of GNRs.

Therefore, further investigation of GNR orientation was performed using polarized light (both p- and s-polarized light) at various tilt angles (Figure 2A). First, we simulated the extinction spectra of perfectly vertically aligned GNRs at various angles from the plane $(\theta)$. According to the results of this simulation, p-polarized light excited both LLSPR and TLSPR when the plane containing the adsorbed GNRs was rotated (Figure 2B). s-Polarized light, however, did not excite LLSPR, even when the plane was rotated (Figure 2C). This tendency was also observed in our experimental data (Figure 2D). In our experimental results, p-polarized light excited LLSPR when the plane was rotated, with the peak LLSPR excitation observed at $620 \mathrm{~nm}$ (Figure 2E). In contrast, LLSPR 
(A)

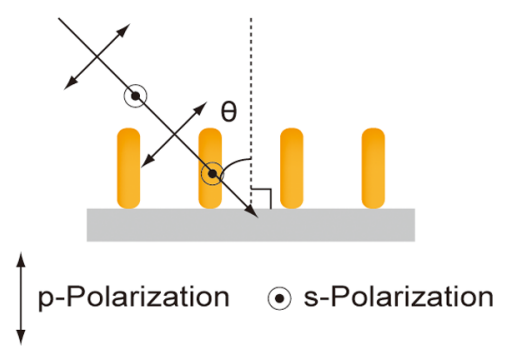

(D)

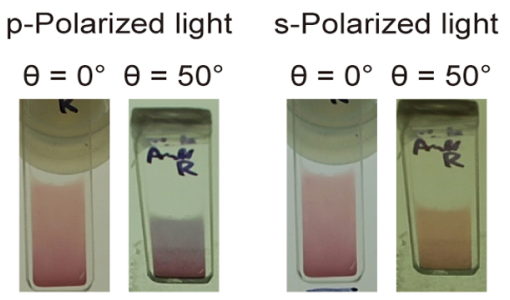

(B)

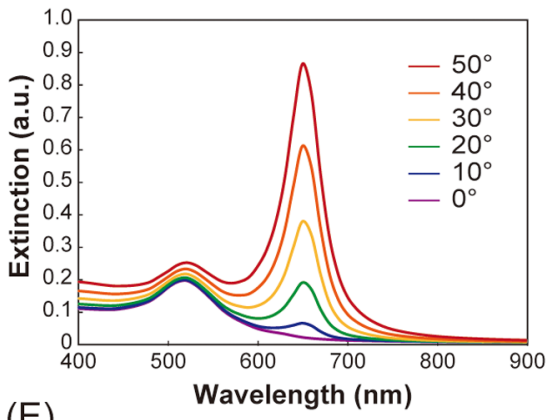

(E)

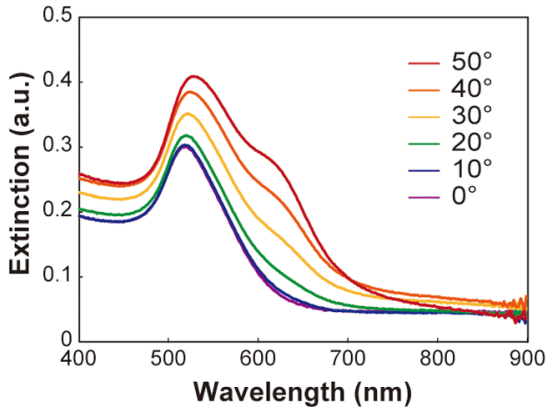

(C)

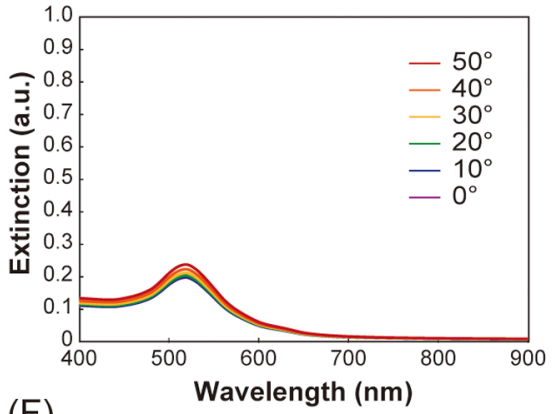

(F)

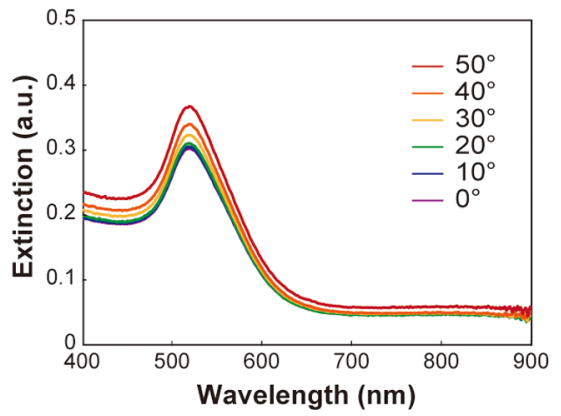

Figure 2. (A) Scheme showing the extinction of GNRs under p- and s-polarized light. Simulated extinction spectra of perfectly aligned GNR under p-polarized (B) and s-polarized (C) light. The center-to-center distance of the GNRs was $30 \mathrm{~nm}$. The peak for the excited LLSPR in (B) is located around $650 \mathrm{~nm}$. (D) Photos of the cuvette containing the adsorbed GNRs through a polarizer. Extinction spectra of GNRs (1:4) adsorbed on a DNA brush (1200 chains $\left./ \mu \mathrm{m}^{2}\right)$ under p-polarized (E) and s-polarized (F) light. The adsorption time was 60 min. The amount of adsorbed GNRs was 2500 particles $/ \mu \mathrm{m}^{2}$. The average center-to-center distance of the adsorbed GNRs was approximately $21 \mathrm{~nm}$. The peak for the excited LLSPR in (E) is located around $620 \mathrm{~nm}$.

(A)

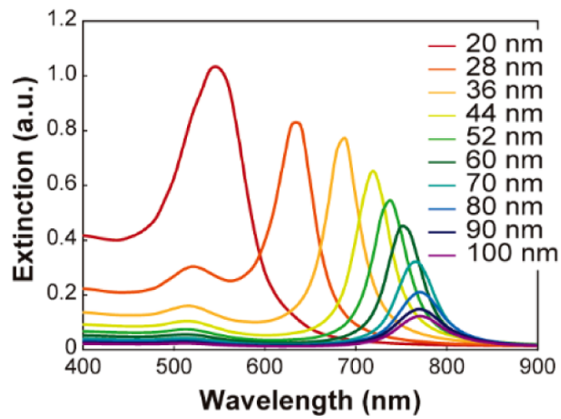

(B)

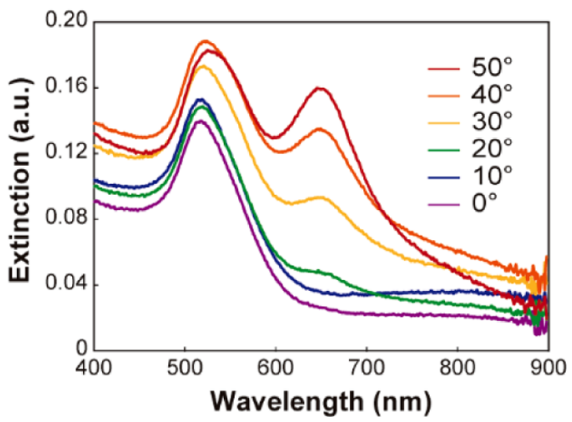

(C)

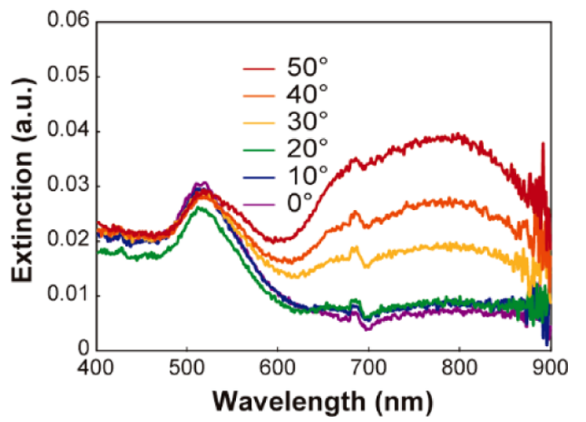

Figure 3. (A) Simulated extinction spectra of perfectly aligned GNRs with various center-to-center distances under p-polarized light at $\theta=50^{\circ}$. (B, C) Extinction spectra of GNRs (1:4) on a DNA brush under p-polarized light. The adsorption time was 10 min (B) or 1 min (C). The center-tocenter distances were calculated as 34 and $62 \mathrm{~nm}$ for 1000 and 300 particles $/ \mu \mathrm{m}^{2}$, respectively. The peaks for the excited LLSPR in (B) and (C) are located around 650 and $780 \mathrm{~nm}$.

was not excited under s-polarized light even when the plane was rotated in the same manner (Figure 2F), indicating that the adsorbed GNRs were aligned vertically to the glass surface. The ratio of LLSPR to TLSPR in the simulation results was higher than that in our experimental results, suggesting that there is some fluctuation in GNRs.

We next confirmed the effect of plasmonic coupling among nearest neighboring GNRs. According to the simulation results for interparticle distance dependence, as the center-to-center distance of the aligned GNRs narrowed, the LLSPR excitation gradually blue-shifted due to plasmonic coupling induced by the side-by-side assembly of GNRs (Figure 3A). ${ }^{32,33}$ This peak shift was also observed in our experimental results. The amount of adsorbed GNRs was tuned by changing the adsorption time
$(1,10$, and $60 \mathrm{~min})$, and the extinction spectra of the GNRs at various tilt angles under p-polarized light were recorded. The amounts of adsorbed GNRs were determined as 300, 1000, and 2500 particles $/ \mu \mathrm{m}^{2}$ by inductively coupled plasma atomic emission spectrometry (ICP-AES) for adsorption times of 1 , 10 , and $60 \mathrm{~min}$, wherein the number ratios of GNRs to DNA chains were $0.25,0.83$, and 2.1 , respectively. These values equate to the average center-to-center distances of the adsorbed GNRs of approximately 62, 34, and $21 \mathrm{~nm}$. To correlate average GNR center-to-center distance to the LLSPR peak positions, the simulated LLSPR peak positions were plotted as a function of the simulated GNR center-to-center distance (Figure S4). The experimentally observed LLSPR peak positions were around 780, 650, and $620 \mathrm{~nm}$, and these 
(A)

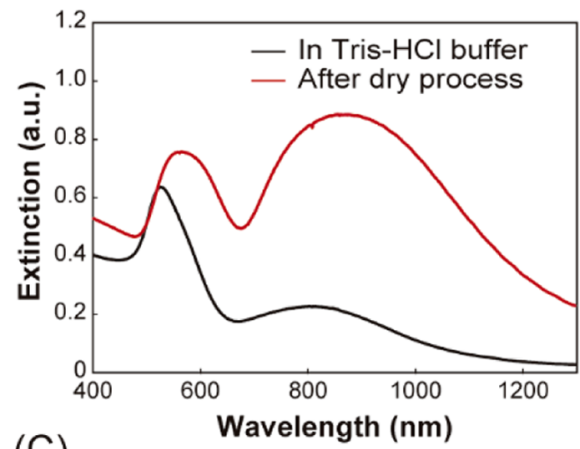

(C)

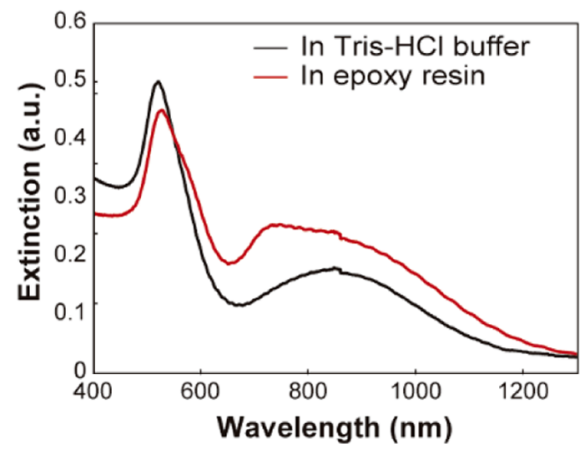

(B)

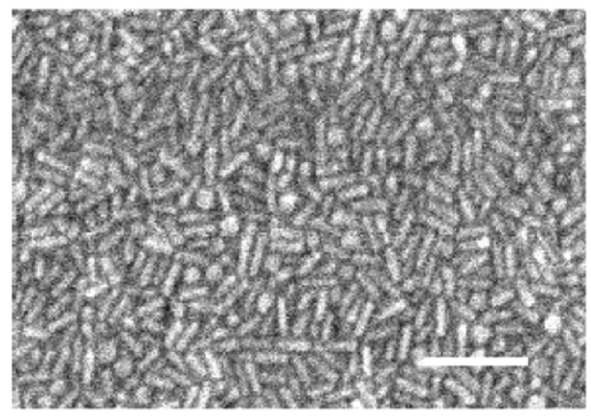

(D)

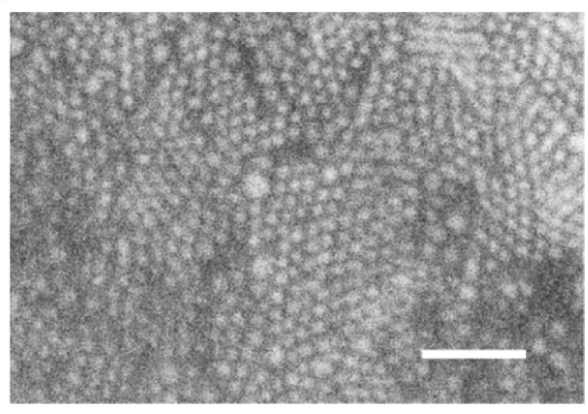

Figure 4. (A) Extinction spectra of GNRs (1:4) on a DNA brush in Tris- $\mathrm{HCl}$ buffer ( $\mathrm{pH}=7.7$ ) and after the drying process. (B) FE-SEM image of the adsorbed GNRs after the drying process. (C) Extinction spectra of the GNRs on a DNA brush before and after the epoxy resin embedding process. (D) FE-SEM image of the adsorbed GNRs in the epoxy resin. The adsorption time was $60 \mathrm{~min}$. The scale bar represents $100 \mathrm{~nm}$ in both cases.

positions corresponded to over 70, 30, and $26 \mathrm{~nm}$, respectively, on the basis of the correlation curve calculated from the simulation. These values obtained from the extinction spectra were also consistent with the values obtained from ICP-AES experiments. Further, both the experimental results and simulation data showed that this vertical alignment was independent of the amount of adsorbed GNRs.

To directly confirm the GNR (1:4) orientation on the DNA brush, field emission scanning electron microscopy (FE-SEM) was performed. In our first attempt to image the orientation of the GNRs, the sample was simply dried and then imaged. However, this approach revealed a strong peak for LLSPR, indicating that the GNRs did not align vertically (Figure 4A). The FE-SEM image also showed that their orientation was random (Figure 4B). The highly oriented GNR structure had become more disordered during the drying process. This structural change was thought to be caused by a lateral capillary force during evaporation of the solvent and suggested that sufficient free space existed around each GNR for them to lay flat. Therefore, to avoid any structural changes induced by the drying process, we employed an epoxy resin to stabilize the GNRs. The GNRs were first embedded in the epoxy resin, which was subsequently decoupled from the glass cuvette after solidification, and then imaged by FE-SEM. Although the extinction spectra subtly changed before and after the epoxy resin embedding process (Figure $4 \mathrm{C}$ ), we observed the GNRs to be round (Figure 4D). The diameter of the round GNRs is similar to that of their minor axis, as shown in Figure 4B, indicating that the GNRs were generally aligned vertically.

On the basis of the above alignment, we believed that the charge density on the GNRs affects their orientation as highly charged GNRs can bend the DNA chains, leading to a random
GNR orientation on the DNA brush. Recently, Yingling and coworkers have simulated the conformational change of $30 \mathrm{bp}$ DNA induced by interaction with positively charged spherical nanoparticles, with a diameter of $\sim 1.5 \mathrm{~nm} .{ }^{34}$ According to their simulations, a high charge density on the nanoparticles bends the DNA, and a low charge density on the nanoparticles allows the nanoparticles to attach to the DNA without bending. Although the DNA length and nanoparticle size and shape in our experiment differed from those in the simulation, our results can be similarly explained by the presence or absence of DNA brush bending. In other words, the grafted DNA bends at the curved surface of the highly cationic GNRs, leading to a random orientation as the grafted DNA is no longer perfectly aligned. In contrast, when GNRs with a low charge density were used, the grafted DNA did not bend and the GNRs were absorbed unidirectionally along the axis of the extended DNA brush.

\section{CONCLUSIONS}

In conclusion, we have demonstrated that the orientation of GNRs can be controlled with the assistance of a dsDNA brush. This was achieved by the simple electrostatics adsorption of positively charged GNRs onto the DNA brush. Interestingly, the density of the positive charge on the surface of the GNRs is important to their vertical alignment. Our approach not only removes limits to the area of ordered GNRs in theoretical terms but also provides a number of possibilities for the control of metal nanorod orientation in more complex systems; for example, the inclusion of colloidal particles on a nonflat surface. Furthermore, by tuning the correlation between the length of the DNA chains on the brush and the size of the metal nanorods, hierarchical ordering of metal nanorods to create and 
tailor multifunctional systems may be achieved. Our research provides new insights into the self-assembly of anisotropic nanoparticles utilizing polymer configuration and electrostatic attraction.

\section{EXPERIMENTAL SECTION}

Synthesis of GNRs. (1) Preparation of the seed solution: $\mathrm{HAuCl}_{4} \cdot 3 \mathrm{H}_{2} \mathrm{O}(250 \mu \mathrm{L}, 0.01 \mathrm{M})$ was added to a CTAB aqueous solution $(7.5 \mathrm{~mL}, 0.1 \mathrm{M})$ in a $50 \mathrm{~mL}$ plastic tube. An ice-cold $\mathrm{NaBH}_{4}$ solution $(600 \mu \mathrm{L}, 0.01 \mathrm{M})$ was injected while the plastic tube was shaken gently. The solution was then left undisturbed for $2 \mathrm{~h}$ at $30{ }^{\circ} \mathrm{C}$ prior to use. (2) Growth reaction: $\mathrm{HAuCl}_{4} \cdot 3 \mathrm{H}_{2} \mathrm{O}(400 \mu \mathrm{L}, 0.01 \mathrm{M}), \mathrm{AgNO}_{3}(240 \mu \mathrm{L}, 0.01 \mathrm{M})$, and ascorbic acid $(64 \mu \mathrm{L}, 0.1 \mathrm{M})$ were added to a CTAB aqueous solution $(40 \mathrm{~mL}, 0.1 \mathrm{M})$ in a $100 \mathrm{~mL}$ plastic tube in the above order, with subsequent injection of the seed solution $(96 \mu \mathrm{L})$. The growth solution was then shaken gently and left undisturbed for $3 \mathrm{~h}$ at $30{ }^{\circ} \mathrm{C}$. (3) Centrifugal purification: The synthesized GNRs solutions $(1.5 \mathrm{~mL})$ were transferred to a 1.7 $\mathrm{mL}$ plastic tube. The GNR solution was concentrated 10-fold by centrifugation $\left(18000 \mathrm{~g}, 20 \mathrm{~min}, 30^{\circ} \mathrm{C}\right)$. Thereafter, all centrifugation was performed under the same conditions. The GNR solutions were centrifuged, and the supernatant (1.45 $\mathrm{mL})$ was removed. The residue $(50 \mu \mathrm{L})$ was diluted with MilliQ $(1.45 \mathrm{~mL})$. This purification procedure was repeated twice.

Surface Exchange of GNRs. Different amounts of two kinds of alkanethiol ligands (the $\mathrm{NH}_{2} \mathrm{EG} 6 \mathrm{C} 11$ and EG6C11 ligand) were mixed in methanol. The ratios of the ligands were $5: 0,4: 1,3: 2,2: 3$, and $1: 4$ in a total alkanethiol ligand concentration of $5 \mathrm{mM}$. The mixed alkanethiol ligand solution (50 $\mu \mathrm{L})$ was added to the purified GNR solution $(1.45 \mu \mathrm{L})$. The mixed solution was purified by two cycles of centrifugation, as described above. Finally, the mixed ligand GNR solution was concentrated to $25 \mathrm{nM}$, as determined by the ICP-AES measurement.

Preparation of Streptavidin-Coated Substrates. A glass cuvette was cleaned with piranha solution (a 3:1 mixture of concentrated $\mathrm{H}_{2} \mathrm{SO}_{4}$ with $\mathrm{H}_{2} \mathrm{O}_{2}$ ) for $1 \mathrm{~h}$, with subsequent rinsing in MilliQ and dried in an incubator $\left(60{ }^{\circ} \mathrm{C}\right)$. The cleaned glass cuvette was placed in a $\mathrm{N}_{2}$-filled glovebox, and the inside surface of the cuvette was immersed in $0.01 \% \mathrm{v} / \mathrm{v}$ superdehydrated toluene of CMETS. A silane coupling reaction was performed for $1 \mathrm{~h}$, followed by rinsing with acetone, ethanol, and MilliQ. After washing, the surface methoxy group was hydrolyzed by treatment with concentrated $\mathrm{HCl}$ for $16 \mathrm{~h}$. To immobilize the biotin, the hydrolyzed glass cuvette was soaked in a $10 \mathrm{mM}$ HEPES-KOH buffer solution $(\mathrm{pH}=7.4)$ containing EDC $(50 \mathrm{mM})$ and amine-PEG - -biotin $(1 \mathrm{mM})$ for $1 \mathrm{~h}$. Finally, streptavidin was immobilized onto the glass cuvette via streptavidin-biotin interaction by incubating the biotinylated surface with a $\mathrm{pH} 7.7$ Tris- $\mathrm{HCl}$ buffer containing streptavidin $(100 \mu \mathrm{g} / \mathrm{mL})$ for $1 \mathrm{~h}$.

PCR Reaction for the Synthesis of 148 bp DNA. For 148 bp DNA brush preparation, 148 bp DNA was synthesized by a standard PCR method using $\lambda$-DNA as a template and a pair of $5^{\prime}$-modified primers ( 22 base biotin primer and 21 base Alexa 647 primer). 22 base biotin primer $(10 \mu \mathrm{M}), 21$ base alexa 647 primer $(10 \mu \mathrm{M})$, and $\lambda$-DNA $(0.20 \mu \mathrm{g} / \mu \mathrm{L})$ were mixed in a ratio of $1: 1: 1$. Then, the primer-template mixed solution, MilliQ and Quick Taq HS DyeMix were mixed at a ratio of 3:22:25. Thermal cycling was carried out as follows: for $2 \mathrm{~min}$ at $94{ }^{\circ} \mathrm{C}$, followed by 20 cycles of $20 \mathrm{~s}$ at $94{ }^{\circ} \mathrm{C}, 30 \mathrm{~s}$ at
$58{ }^{\circ} \mathrm{C}$, and $1 \mathrm{~min}$ at $68{ }^{\circ} \mathrm{C}$. After the reaction, the products were cooled to $4{ }^{\circ} \mathrm{C}$. The products were purified using a Qiagen PCR purification kit to remove the nonpolymerized primers.

Preparation of a DNA Brush and Adsorption of GNRs. The synthesized 148 bp DNA was immobilized by soaking the streptavidin-coated cuvette in a $\mathrm{pH} 7.7$ Tris- $\mathrm{HCl}$ buffer containing $100 \mathrm{nM}$ DNA and $1000 \mathrm{mM} \mathrm{NaCl}$. The GNRs modified with the alkanethiol ligands were attached by soaking the inside surface of the DNA brush cuvettes in the GNR solution. After that, the inside surface of the cuvette was washed with $\mathrm{pH} 7.7$ Tris- $\mathrm{HCl}$ at least three times to remove the nonattached GNRs.

Finite Difference Time Domain (FDTD) Calculation. We estimated the optical properties of the perfectly aligned GNR structure using an electromagnetic optical simulation on the basis of the FDTD method. The FDTD method solves Maxwell's equations by discretizing the equations via central differences in time and space and then numerically solving these equations using software. For simplicity, we have assumed that the GNRs were arranged in a hexagonal pattern. The effect of the solvent has been accounted for by the refractive index of the ambient environment, that is, $n=1.333$ for water.

Quantitative Analysis of the Amount of Adsorbed GNRs. The adsorbed GNRs were dissolved with aqua regia $\left(\mathrm{HNO}_{3} / \mathrm{HCl}=1: 3\right)$. The amount of gold contained in the aqua regia was quantitatively analyzed by ICP-AES. For simplicity, we considered the GNRs $(34 \mathrm{~nm} \times 10 \mathrm{~nm})$ as cylinders and estimated the density of the adsorbed GNRs on the DNA brush surface.

Embedding of the Adsorbed GNRs in Epoxy Resin. EPON812 RESIN, DDSA, and MNA were mixed in a ratio of 4.6, 2.6, and 2.8, and subsequently stirred for $20 \mathrm{~min}$. DMP-30 was then added into the mixed solution. After stirring for 60 $\mathrm{min}$, the resin solution was degassed under vacuum for $1 \mathrm{~h}$. The mixture was applied on the adsorbed GNRs and left at $60{ }^{\circ} \mathrm{C}$ for 3 days for solidification. The solidified epoxy resin was decoupled from the cuvette and coated with carbon (10-20 $\mathrm{nm})$.

\section{ASSOCIATED CONTENT}

\section{Supporting Information}

The Supporting Information is available free of charge on the ACS Publications website at DOI: 10.1021/acsomega.7b00303.

Experimental procedures and setups, TEM images, extinction spectra, $\zeta$ potential measurements, and LLSPR peak plots (PDF)

\section{AUTHOR INFORMATION}

\section{Corresponding Authors}

*E-mail: mitomo@poly.es.hokudai.ac.jp (H.M.).

*E-mail: ijiro@poly.es.hokudai.ac.jp (K.I.).

ORCID

Hideyuki Mitomo: 0000-0002-6748-7816

Kuniharu Ijiro: 0000-0003-0488-765X

\section{Present Address}

${ }^{\nabla}$ Department of Innovative Systems Engineering, and Graduate School of Environmental Symbiotic System Major, Nippon Institute of Technology, Miyashiro, Saitama 345-8501, Japan (K.N.). 


\section{Notes}

The authors declare no competing financial interest.

\section{ACKNOWLEDGMENTS}

This work was supported in part by the "Dynamic Alliance for Open Innovation Bridging Human, Environment and Materials" from the Ministry of Education, Culture, Sports, Science, and Technology of Japan (MEXT). This work was performed under the Cooperative Research Program of the "Network Joint Research Center for Materials and Devices". A part of this work was conducted at Hokkaido University, supported by the "Nanotechnology Platform" Program of the MEXT, Japan. We thank the OPEN FACILITY of Hokkaido University Sousei Hall for providing access to the Delsa Nano HC system and ICPE-9000. We also thank the Material Analysis and Structure Analysis Open Unit (MASAOU) of Hokkaido University for allowing the use of the UV-vis spectrometer (Lambda 900S).

\section{REFERENCES}

(1) Rosi, N. L.; Mirkin, C. A. Nanostructures in Biodiagnostics. Chem. Rev. 2005, 105, 1547-1562.

(2) Stewart, M. E.; Anderton, C. R.; Thompson, L. B.; Maria, J.; Gray, S. K.; Rogers, J. A.; Nuzzo, R. G. Nanostructured Plasmonic Sensors. Chem. Rev. 2008, 108, 494-521.

(3) Mitomo, H.; Horie, K.; Matsuo, Y.; Niikura, K.; Tani, T.; Naya, M.; Ijiro, K. Active Gap SERS for the Sensitive Detection of Biomacromolecules with Plasmonic Nanostructures on Hydrogels. Adv. Opt. Mater. 2016, 4, 259-263.

(4) Ghosh, P.; Han, G.; De, M.; Kim, C. K.; Rotello, V. M. Gold nanoparticles in delivery applications. Adv. Drug Delivery Rev. 2008, 60, 1307-1315.

(5) Niikura, K.; Iyo, N.; Matsuo, Y.; Mitomo, H.; Ijiro, K. Sub-100 nm Gold Nanoparticle Vesicles as a Drug Delivery Carrier enabling Rapid Drug Release upon Light Irradiation. ACS Appl. Mater. Interfaces 2013, 5, 3900-3907.

(6) Linic, S.; Aslam, U.; Boerigter, C.; Morabito, M. Photochemical transformations on plasmonic metal nanoparticles. Nat. Mater. 2015, $14,567-576$.

(7) Wang, G.; Mitomo, H.; Matsuo, Y.; Shimamoto, N.; Niikura, K.; Ijiro, $\mathrm{K}$. DNA-templated plasmonic $\mathrm{Ag} / \mathrm{AgCl}$ nanostructures for molecular selective photocatalysis and photocatalytic inactivation of cancer cells. J. Mater. Chem. B 2013, 1, 5899-5907.

(8) Xu, L.; Ma, W.; Wang, L.; Xu, C.; Kuang, H.; Kotov, N. A. Nanoparticle assemblies: dimensional transformation of nanomaterials and scalability. Chem. Soc. Rev. 2013, 42, 3114-3126.

(9) Nie, Z.; Petukhova, A.; Kumacheva, E. Properties and emerging applications of self-assembled structures made from inorganic nanoparticles. Nat. Nanotechnol. 2010, 5, 15-25.

(10) Fan, J. A.; Wu, C.; Bao, K.; Bao, J.; Bardhan, R.; Halas, N. J.; Manoharan, V. N.; Nordlander, P.; Shvets, G.; Capasso, F. SelfAssembled Plasmonic Nanoparticle Clusters. Science 2010, 328, 11351138.

(11) Niikura, K.; Iyo, N.; Higuchi, T.; Nishio, T.; Jinnai, H.; Fujitani, N.; Ijiro, K. Gold Nanoparticles Coated with Semi-Fluorinated Oligo(ethylene glycol) Produce Sub-100 nm Nanoparticle Vesicles without Templates. J. Am. Chem. Soc. 2012, 134, 7632-7635.

(12) Wei, J.; Niikura, K.; Higuchi, T.; Kimura, T.; Mitomo, H.; Jinnai, H.; Joti, Y.; Bessho, Y.; Nishino, Y.; Matsuo, Y.; et al. Yolk/Shell Assembly of Gold Nanoparticles by Size Segregation in Solution. J. Am. Chem. Soc. 2016, 138, 3274-3277.

(13) Huang, X.; El-Sayed, M. A. Gold nanoparticles: Optical properties and implementations in cancer diagnosis and photothermal therapy. J. Adv. Res. 2010, 1, 13-28.

(14) Liz-Marzán, L. M. Nanometals: Formation and color. Mater. Today 2004, 7, 26-31.
(15) Baker, J. L.; Widmer-Cooper, A.; Toney, M. F.; Geissler, P. L.; Alivisatos, A. P. Device-Scale Perpendicular Alignment of Colloidal Nanorods. Nano Lett. 2010, 10, 195-201.

(16) Singh, A.; Gunning, R. D.; Ahmed, S.; Barrett, C. A.; English, N. J.; Garate, J.-A.; Ryan, K. M. Controlled semiconductor nanorod assembly from solution: influence of concentration, charge and solvent nature. J. Mater. Chem. 2012, 22, 1562-1569.

(17) Ryan, K. M.; Mastroianni, A.; Stancil, K. A.; Liu, H.; Alivisatos, A. P. Electric-Field-Assisted Assembly of Perpendicularly Oriented Nanorod Superlattices. Nano Lett. 2006, 6, 1479-1482.

(18) Gupta, S.; Zhang, Q.; Emrick, T.; Russell, T. P. "Self-Corralling" Nanorods under an Applied Electric Field. Nano Lett. 2006, 6, 20662069.

(19) Hu, Z.; Fischbein, M. D.; Querner, C.; Drndić, M. ElectricField-Driven Accumulation and Alignment of CdSe and CdTe Nanorods in Nanoscale Devices. Nano Lett. 2006, 6, 2585-2591.

(20) Carbone, L.; Nobile, C.; De Giorgi, M.; Sala, F. D.; Morello, G.; Pompa, P.; Hytch, M.; Snoeck, E.; Fiore, A.; Franchini, I. R.; et al. Synthesis and Micrometer-Scale Assembly of Colloidal CdSe/CdS Nanorods Prepared by a Seeded Growth Approach. Nano Lett. 2007, 7, 2942-2950.

(21) Kim, K.; Han, H. S.; Choi, I.; Lee, C.; Hong, S.; Suh, S.-H.; Lee, L. P.; Kang, T. Interfacial liquid-state surface-enhanced Raman spectroscopy. Nat. Commun. 2013, 4, No. 2182.

(22) Kim, F.; Kwan, S.; Akana, J.; Yang, P. Langmuir-Blodgett Nanorod Assembly. J. Am. Chem. Soc. 2001, 123, 4360-4361.

(23) Flauraud, V.; Mastrangeli, M.; Bernasconi, G. D.; Butet, J.; Alexander, D. T. L.; Shahrabi, E.; Martin, O. J. F.; Brugger, J. Nanoscale topographical control of capillary assembly of nanoparticles. Nat. Nanotechnol. 2016, 12, 73-80.

(24) Thai, T.; Zheng, Y.; Ng, S. H.; Mudie, S.; Altissimo, M.; Bach, U. Self-Assembly of Vertically Aligned Gold Nanorod Arrays on Patterned Substrates. Angew. Chem., Int. Ed. 2012, 51, 8732-8735.

(25) Bracha, D.; Karzbrun, E.; Shemer, G.; Pincus, P. A.; Bar-Ziv, R. H. Entropy-driven collective interactions in DNA brushes on a biochip. Proc. Natl. Acad. Sci. U.S.A. 2013, 110, 4534-4538.

(26) Netz, R. R.; Andelman, D. Neutral and charged polymers at interfaces. Phys. Rep. 2003, 380, 1-95.

(27) Das, S.; Banik, M.; Chen, G.; Sinha, S.; Mukherjee, R. Polyelectrolyte brushes: theory, modelling, synthesis and applications. Soft Matter 2015, 11, 8550-8583.

(28) Tokarev, I.; Minko, S. Tunable plasmonic nanostructures from noble metal nanoparticles and stimuli-responsive polymers. Soft Matter 2012, 8, 5980-5987.

(29) Tokareva, I.; Minko, S.; Fendler, J. H.; Hutter, E. Nanosensors Based on Responsive Polymer Brushes and Gold Nanoparticle Enhanced Transmission Surface Plasmon Resonance Spectroscopy. J. Am. Chem. Soc. 2004, 126, 15950-15951.

(30) Sau, T. K.; Murphy, C. J. Seeded High Yield Synthesis of Short $\mathrm{Au}$ Nanorods in Aqueous Solution. Langmuir 2004, 20, 6414-6420.

(31) Fu, L.; Liu, Y.; Wang, W.; Wang, M.; Bai, Y.; Chronister, E. L.; Zhen, L.; Yin, Y. A pressure sensor based on the orientational dependence of plasmonic properties of gold nanorods. Nanoscale 2015, 7, 14483-14488.

(32) Gluodenis, M.; Foss, C. A. The Effect of Mutual Orientation on the Spectra of Metal Nanoparticle Rod-Rod and Rod-Sphere Pairs. J. Phys. Chem. B 2002, 106, 9484-9489.

(33) Jain, P. K.; Eustis, S.; El-Sayed, M. A. Plasmon Coupling in Nanorod Assemblies: Optical Absorption, Discrete Dipole Approximation Simulation, and Exciton-Coupling Model. J. Phys. Chem. B 2006, 110, 18243-18253.

(34) Nash, J. A.; Singh, A.; Li, N. K.; Yingling, Y. G. Characterization of Nucleic Acid Compaction with Histone-Mimic Nanoparticles through All-Atom Molecular Dynamics. ACS Nano 2015, 9, 1237412382 . 